\title{
LISA Laser System and European Development Strategy
}

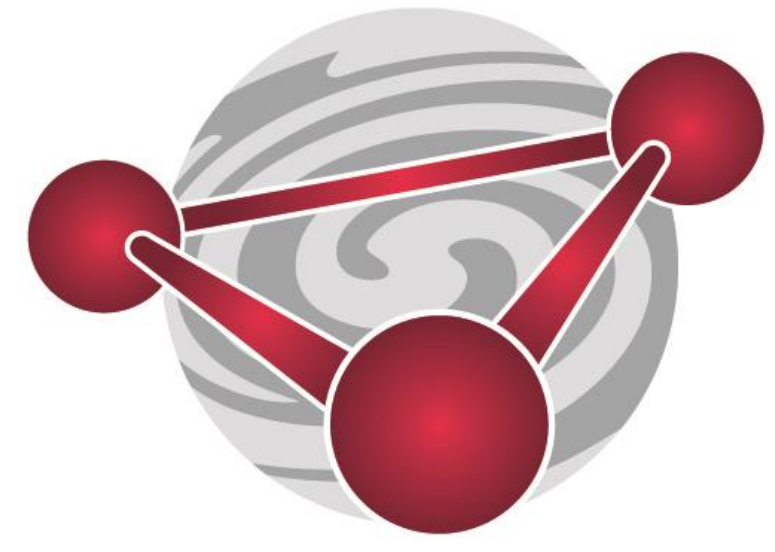

lisa

L. Mondin for the LISA study team

ICSO 2018 09/10/2018

॥ー 


\section{Outline}

- Gravitational Waves and their observation... in space: the LISA observatory

Deadly precision... to look at black holes from the dawn of the universe

- Laser interferometry... over millions of $\mathrm{km}$ ?

- Frightening requirements ... With lots of careful thinking

口 ESA Laser system development strategy

$\square$ Summary 
- LISA - the Laser Interferometer Space Antenna - is a European Space Agency mission to observe the low frequency gravitational waves from space

- LISA has been around for over 20 years, with a science case which has remained stable over the years. However, there was always doubts that:

1. Gravitational waves do not exist!

2. Even if they did, we'd not be capable of building an instrument sensitive enough to measure the miniscule effect of a passing $\mathrm{GW}$, let alone do so in space...

- Then came the second half of $2015 \ldots$.

- LIGO makes first direct detection of gravitational waves $\rightarrow 14$ September 2015

- LISA Pathfinder launched $\rightarrow 3$ December 2015

- LISA Pathfinder performance surpasses all expectations

- Both momentous events led the way to the early selection of LISA by the Science Programme Committee (SPC) of ESA in June 2017 


\section{Gravitational waves... what are they?}

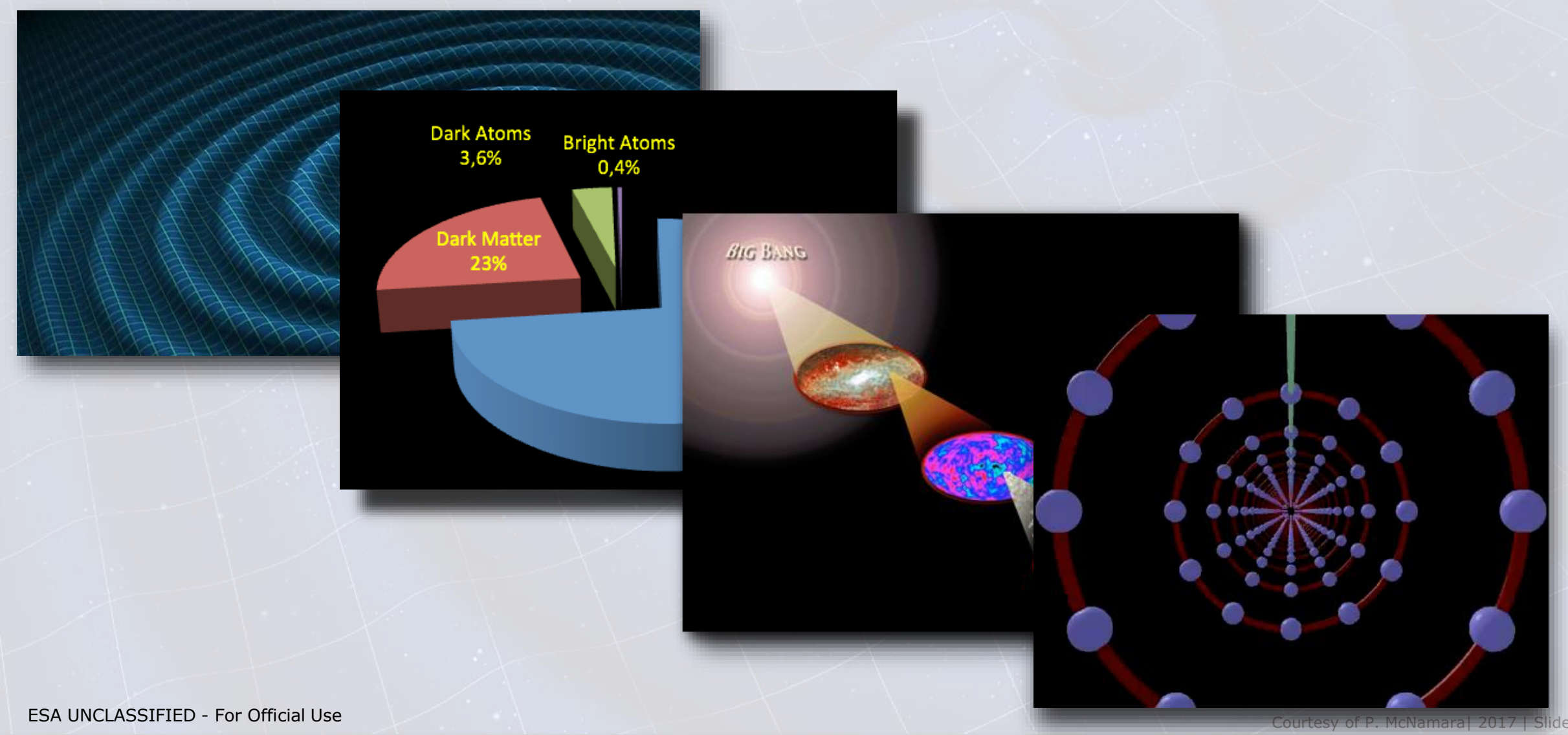

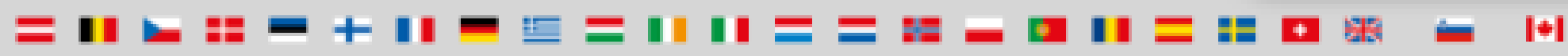




\section{A new way to do astronomy...GW150914}
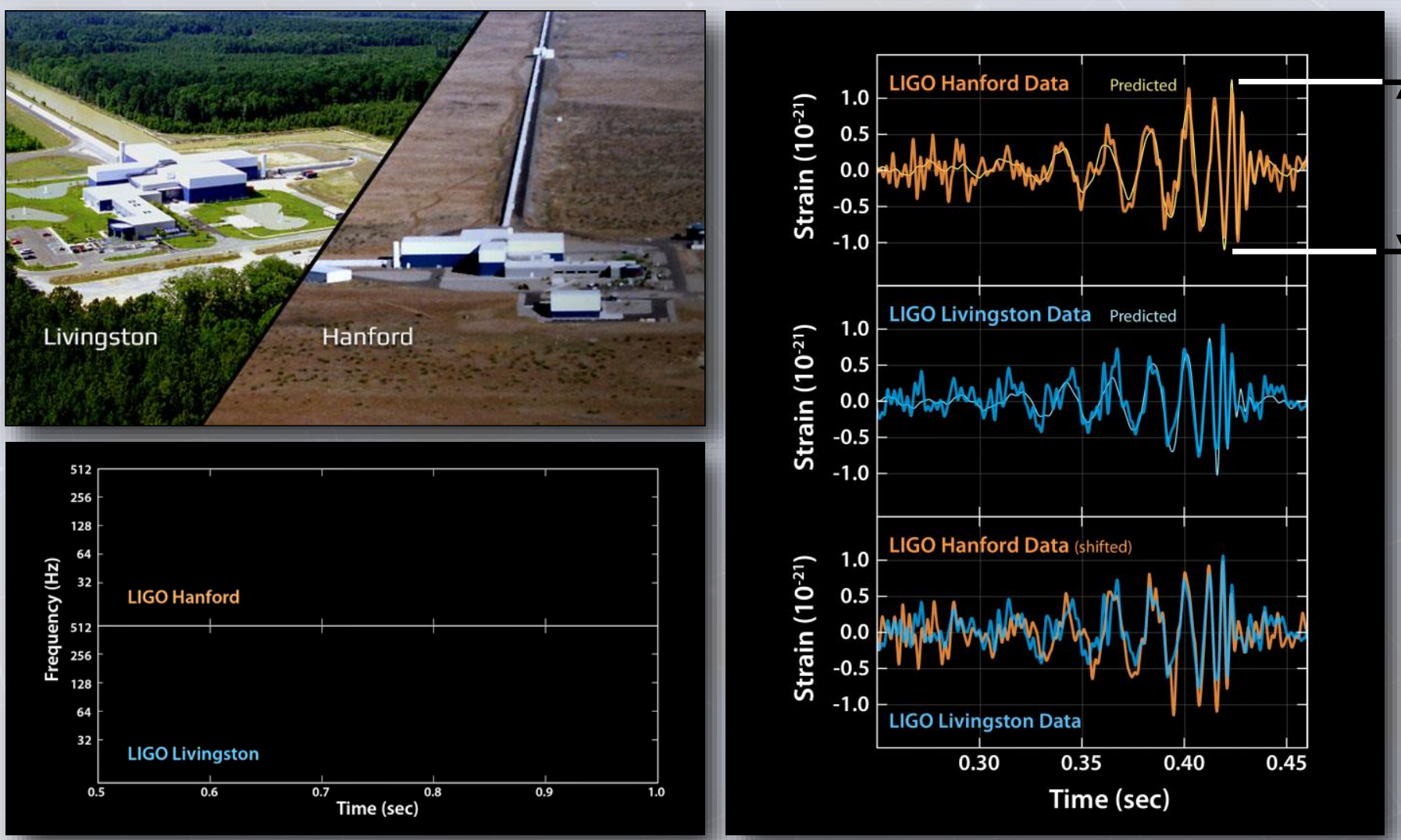

ESA UNCLASSIFIED - For Official Use

[Abbott et al, PRL 2016]

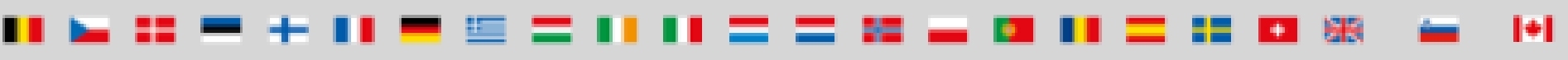


- In October 2017, LIGO-Virgo announced a new discovery....the merger of a pair of Neutron Stars

- For the first time, we also measured the light given off when the stars merged!

- We measured, for the first time.....a kilonova

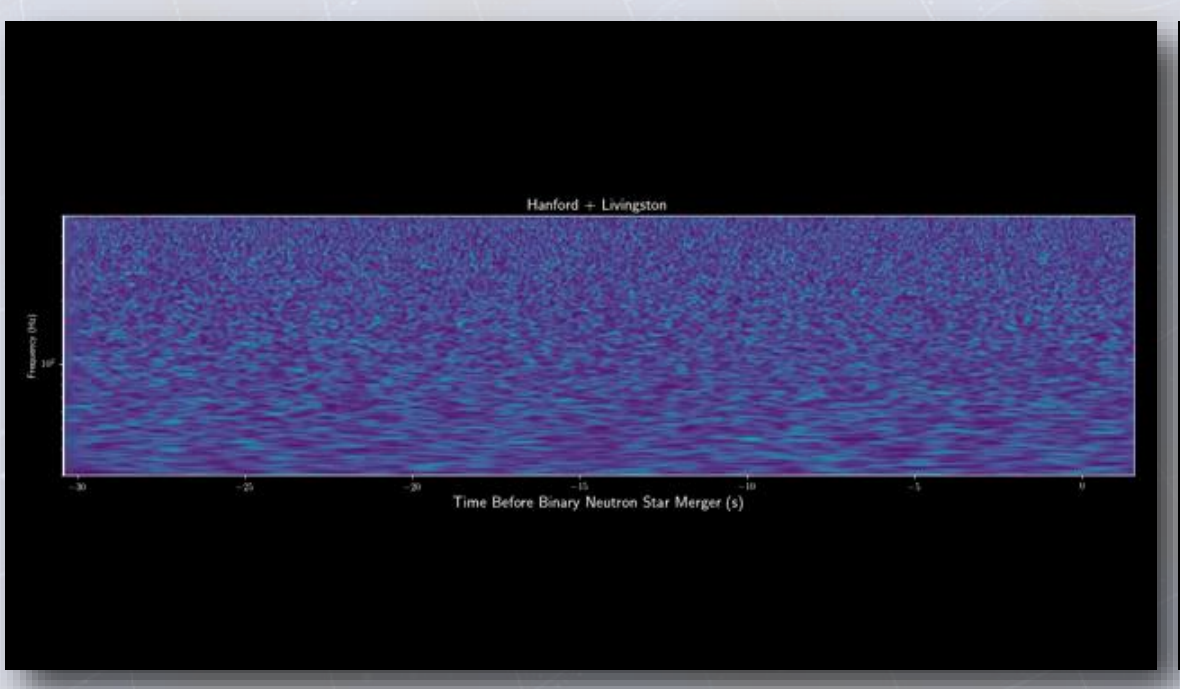

(c) Goddard Space Flight Center

ESA UNCLASSIFIED - For Official Use

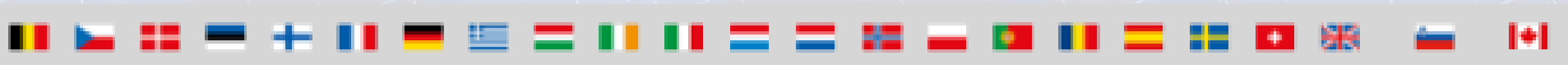




\section{How will we observe GW from space?}

* We essentially build LIGO in space

- But we make it bigger...and call it LISA

- LIGO arms are $4 \mathrm{~km}$ long

* In LISA, the arms will be 2.5 million $\mathrm{km}$ long
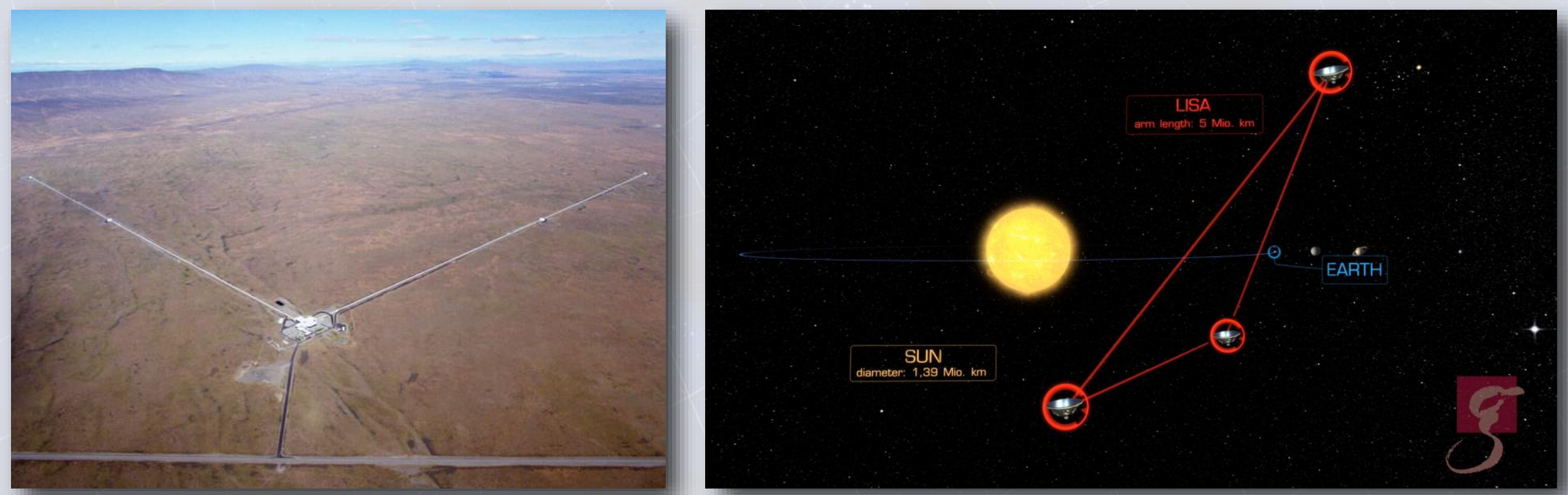

ESA UNCLASSIFIED - For Official Use

uル 
Three spacecraft in an equilateral triangle

$2,500,000 \mathrm{~km}$

Only connection is by laser link

A passing gravitational wave will change length of arms by $\sim 1 \mathrm{pm}$ (rms)... ...equivalent to a change of $160 \mu \mathrm{m}$ (thickness of piece of paper) in the distance to Proxima Centauri 


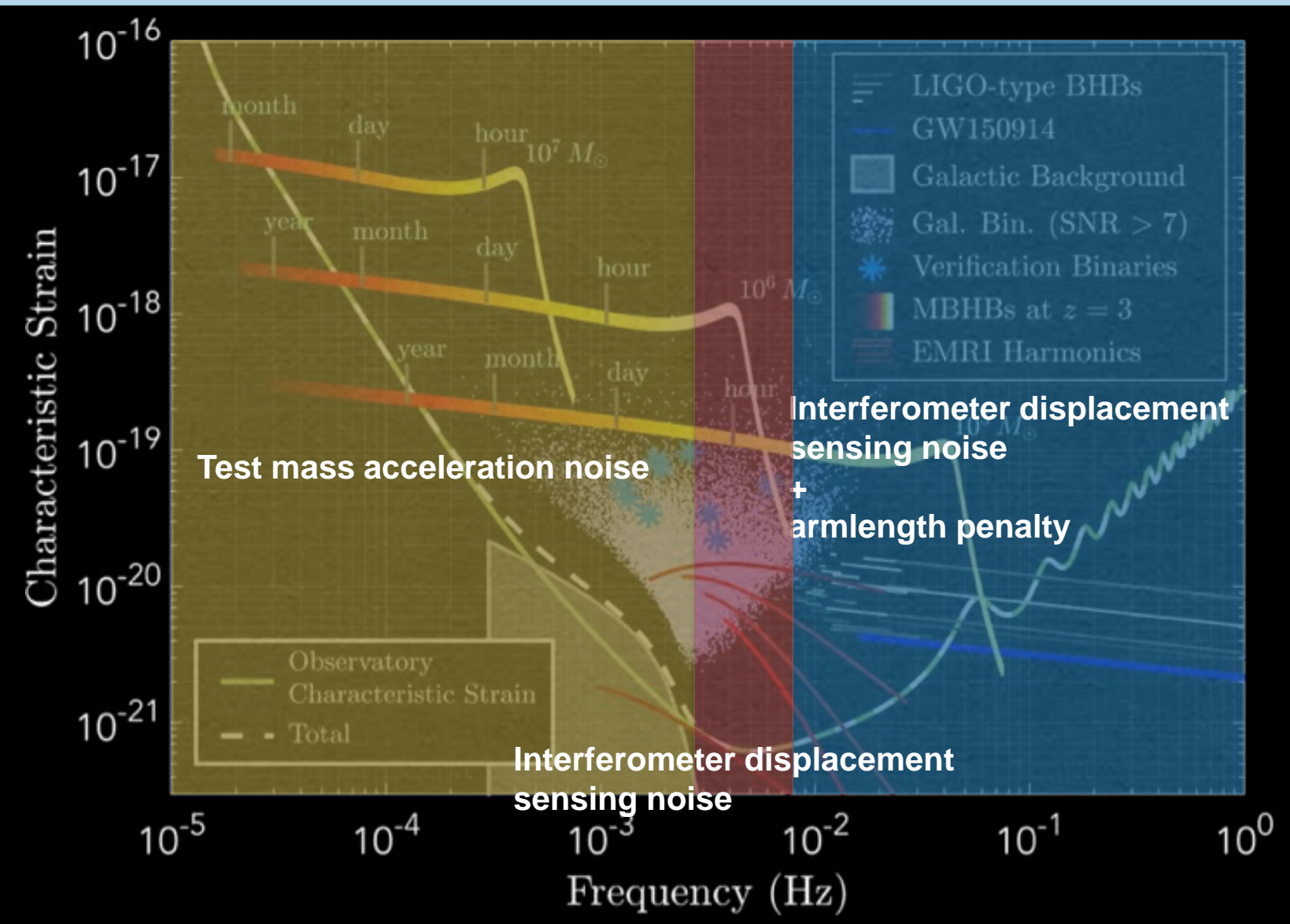

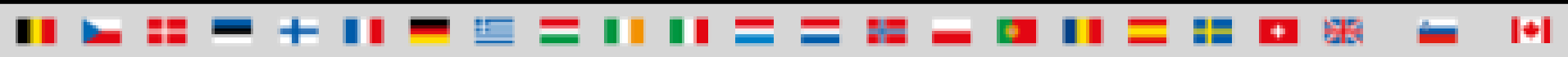




\section{Measurement principle}

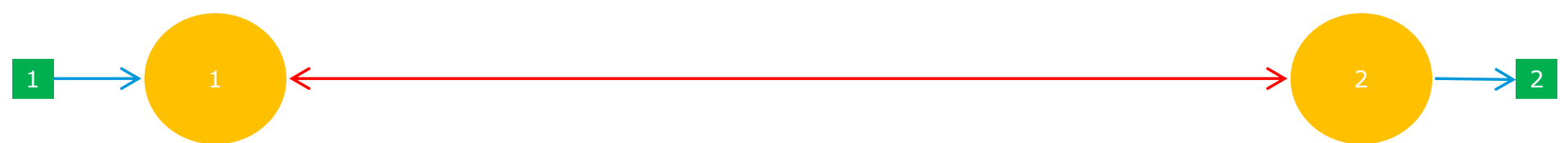

Power loss (divergence of laser beam and limited size of telescopes) implies need to regenerate laser power at second spacecraft.

-Phase lock loop of on-board laser to distant laser

- 1 master laser, 5 slaves

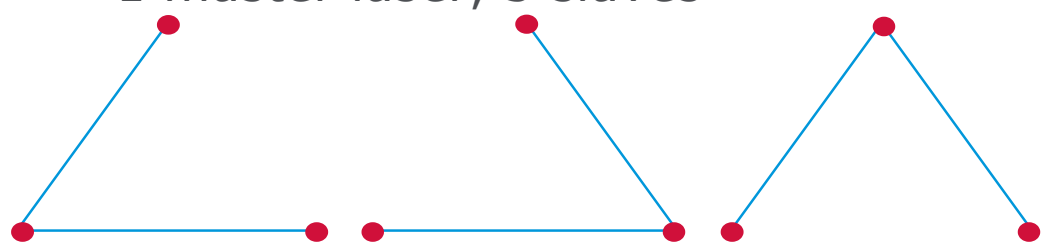

+ Sagnac...

spacecraft A

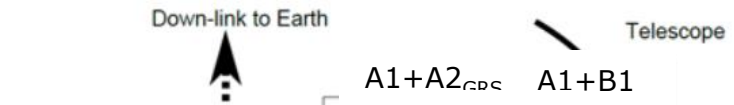

O Optical inter-satellite telecom

$\square$ Synchronisation of clocks

ESA UNCLASSIFIED - For Official Use

Spacecraft A
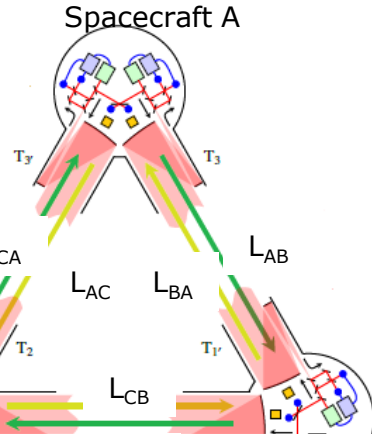

Spacecraft B 


\section{LASER SYSTEM REQUIREMENTS}




\section{LISA Laser System}

- Each S/C has two LA associated with a Moving Optical SubAssembly and pointing toward the distant S/Cs.

- Each LA contains 2 LH Laser Heads (4 LH per S/C, 12 in constellation)

- Cold redundancy

- LOM and LEM

- LOM contains MO and PA

- 1 Laser is master the others are slaves

- 1 LPS per S/C

- Redundancy at constellation level

- Interfaces with LA(s) and/or Phasemeter (TBC)

ESA UNCLASSIFIED - For Official Use

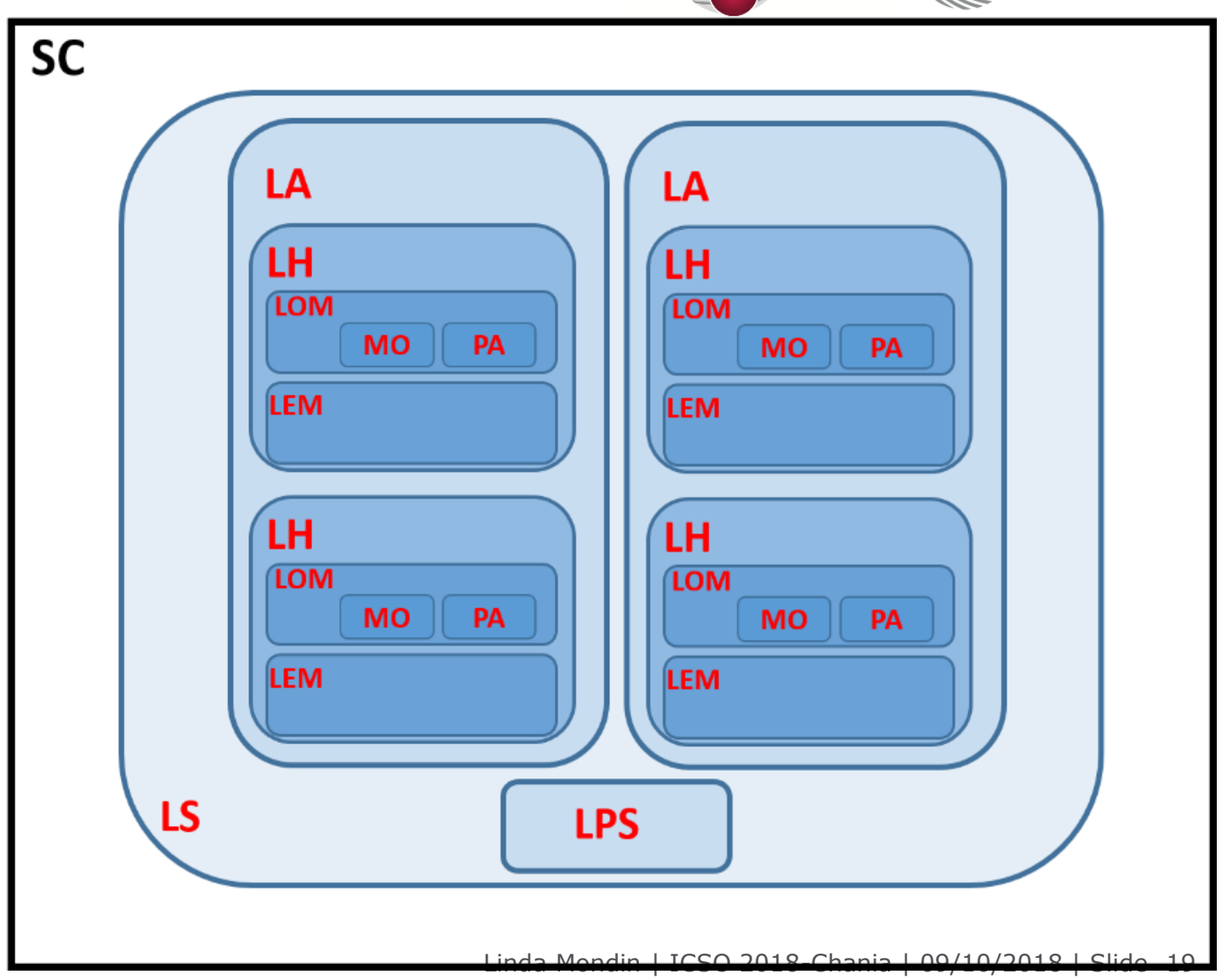




\section{High Level Requirements}

- Dimensions 200x200x200mm per LH.

- Mass $10 \mathrm{~kg}$ per LH

- Volume and mass allocations to be spread between sub-units (LOM and LEM).

- Laser System dissipated power <50W

- LS OP temperature $20 \pm 10^{\circ} \mathrm{C}$ (TBC)

- LS NOP temperature $-20^{\circ} \mathrm{C}$ to $+50^{\circ} \mathrm{C}$ (TBC)

- Laser Electronics Module interfaces TBD depending on concept.

- $>2 \mathrm{~W}$ on $\mathrm{OB}$ at EoL

- Polarisation linear (S TBC)

- Few mW pick-off for LPS

- Lifetime >16years (includes ground testing, cruise, normal and extended science ops)

- Wavelength 1064.049nm

- Laser Pre-Stabilisation 200×200x150mm, 12kg, 6W power consumption. Can consist of two units. 


\section{Frequency requirements (Free running)}

- $\quad$ Frequency stability requirement to succeed phase lock

$S_{v}(f) \leq 3.75 \frac{\mathrm{Hz}}{\sqrt{\mathrm{Hz}}} \sqrt{1+\left(\frac{8 \mathrm{kHz}}{f}\right)^{2}}$ for $3 \times 10^{-5} \mathrm{~Hz} \leq f \leq 1 \mathrm{~Hz}$

and the excess noise requirement for the free-running laser is given by:

$\delta_{p p} v \leq 33 \mathrm{MHz}$ for $3 \times 10^{-5} \mathrm{~Hz} \leq \mathrm{f} \leq 1 \mathrm{~Hz}$

$\delta_{p p} v \leq 200 \mathrm{kHz}$ for $1 \mathrm{~Hz} \leq \mathrm{f} \leq 1 \mathrm{MHz}$ 


\section{Frequency requirements}

- $\quad$ Frequency stability requirement (TDI driven - TBC):

$$
\begin{aligned}
& S_{v}(f) \leq 30 \frac{\mathrm{Hz}}{\sqrt{\mathrm{Hz}}} \times \sqrt{1+\left(\frac{2.8 \mathrm{mHz}}{f}\right)^{4}} \quad \text { For } 3 \cdot 10^{-5} \leq f \leq 1 \mathrm{~Hz} \\
& \delta_{P P}(v) \leq 50 \mathrm{kHz} \\
& S_{v}(f) \leq 3.75 \frac{\mathrm{Hz}}{\sqrt{\mathrm{Hz}}} \times \sqrt{1+\left(\frac{8 \mathrm{kHz}}{f}\right)^{2}} \quad \text { For } 1 \mathrm{~Hz} \leq f \leq 1 \mathrm{MHz} \\
& \delta_{P P}(v) \leq 200 \mathrm{kHz}
\end{aligned}
$$

- Frequency absolute precision 1064.490 nm.

- $\quad$ Frequency reproducibility $\pm 50 \mathrm{MHz}$ (TBC)

- $\quad$ Residual phase error between two lasers $<\sim 2 \mu-$ cycle/ $\sqrt{ }(\mathrm{Hz}) \times \sqrt{ }\left(1+(2.8 \mathrm{mHz} / \mathrm{f})^{4}\right)$ for $3 \mathrm{E}-5<\mathrm{f}<1 \mathrm{~Hz}$

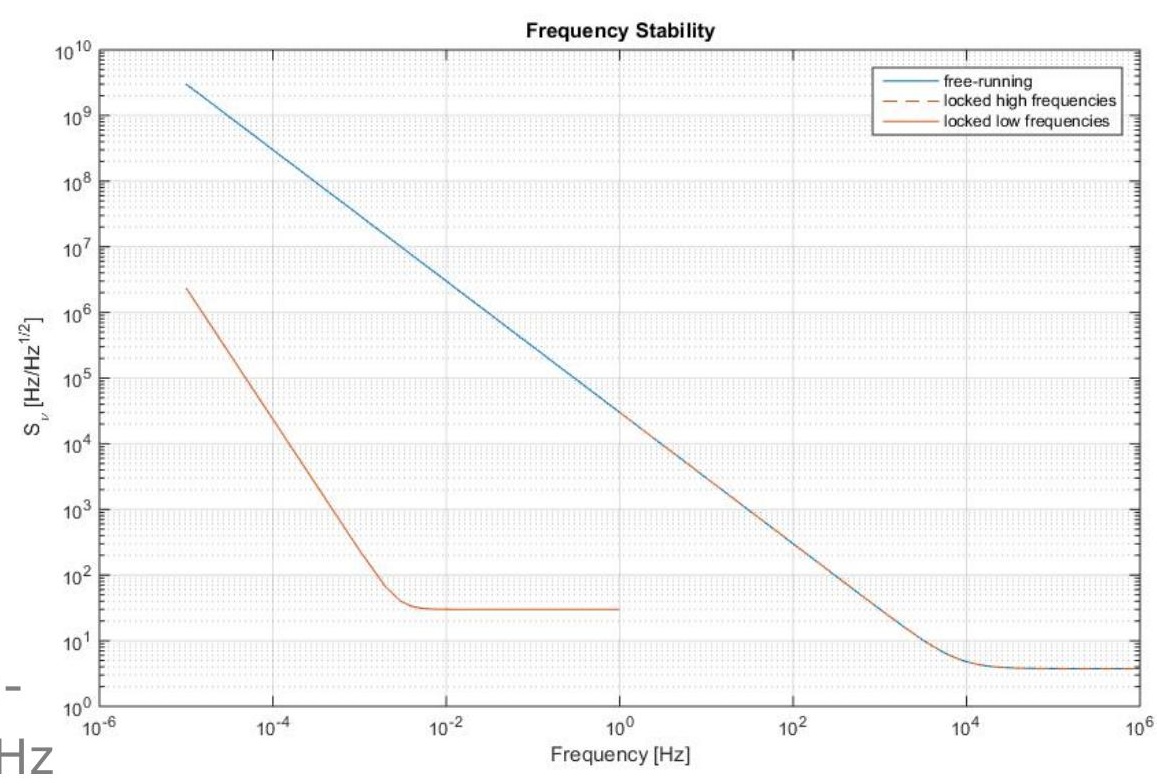

- $\quad$ Differential phase noise (carrier/sideband) 0.6 $\mathrm{mrad} / \sqrt{ } \mathrm{Hz} \times \sqrt{ }\left(1+(2.8 \mathrm{mHz} / \mathrm{f})^{4}\right)$ for $3 \mathrm{E}-5<\mathrm{f}<1 \mathrm{~Hz}$ 


\section{RIN stability requirements}

\section{Free-running laser relative intensity noise (RIN)}

$>10 \mathrm{~mW}$ of optical power is required to show $<10^{-8} / \sqrt{ } \mathrm{Hz}$ (shot-noise level) RIN @ 2 40 MHz range on low noise detector (TBC, heterodyne frequency $\sim 2 x$ heterodyne frequency).

\section{$\square$ Stabilized laser RIN}

The amplifier output must be stabilized to $10^{-4} / \sqrt{ } \mathrm{Hz}$ level at the measurement band, to minimize the classical radiation pressure noise onto the LISA's test masses.

$$
\begin{gathered}
\left.\begin{array}{c}
S_{R I N}(f)<10^{-4} / \sqrt{H z} \\
\delta_{p p} R I N<0.06
\end{array}\right\} \text { for } 3 \times 10^{-5} \mathrm{~Hz} \leq f \leq 10 \mathrm{kHz} \\
\left.\begin{array}{c}
S_{R I N}(f)<10^{-5} / \sqrt{H z} \\
\delta_{p p} R I N<0.02
\end{array}\right\} \text { for } 10 \mathrm{kHz} \leq f \leq 100 \mathrm{kHz} \\
\left.\begin{array}{c}
S_{R I N}(f)<10^{-4} / \sqrt{\mathrm{Hz}} \\
\delta_{p p} R I N<0.02
\end{array}\right\} \text { for } 100 \mathrm{kHz} \leq f \leq 700 \mathrm{kHz} \\
\left.\begin{array}{c}
S_{R I N}(f)<3 \times 10^{-7} / \sqrt{H z} \\
\delta_{p p} R I N<1 \times 10^{-4} \\
S_{R I N}(f)<10^{-8} / \sqrt{H z} \\
\delta_{p p} R I N<5 \times 10^{-4}
\end{array}\right\} \text { for } 700 \mathrm{kHz} \leq f \leq 5 \mathrm{MHz} \\
\text { for } 5 \mathrm{MHz} \leq f \leq 50 \mathrm{MHz}
\end{gathered}
$$




\section{ESA LS DEVELOPMENT STRATEGY}


$>$ LISA PathFinder demonstrated $\mathrm{MO} T R L=9$, TESAT (DE) NPRO

$>$ ESA Laser Optical Communications developments (TESAT (DE)) demonstrated multi Watt MO+PA TRL $=$ 9,

- Performance requirements very
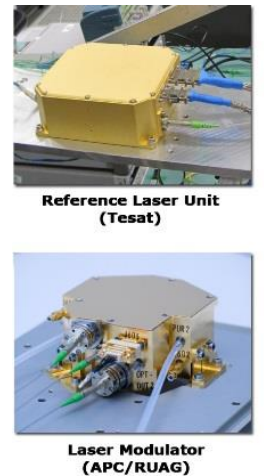

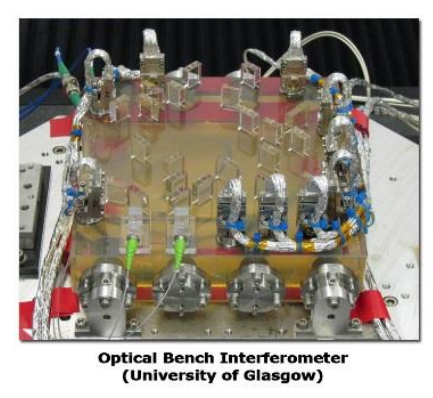

Optical Bench Interferometer
(University of Glasgow)

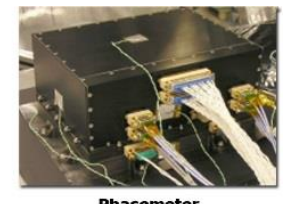

Phasemeter
(University of Birmingham)

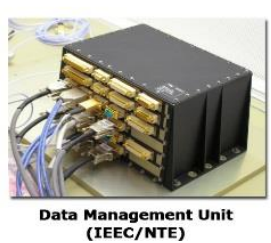
different from LISA $\uparrow$

$>$ ESA Developments:

- MO alternatives

- PA + LEM

- LPS

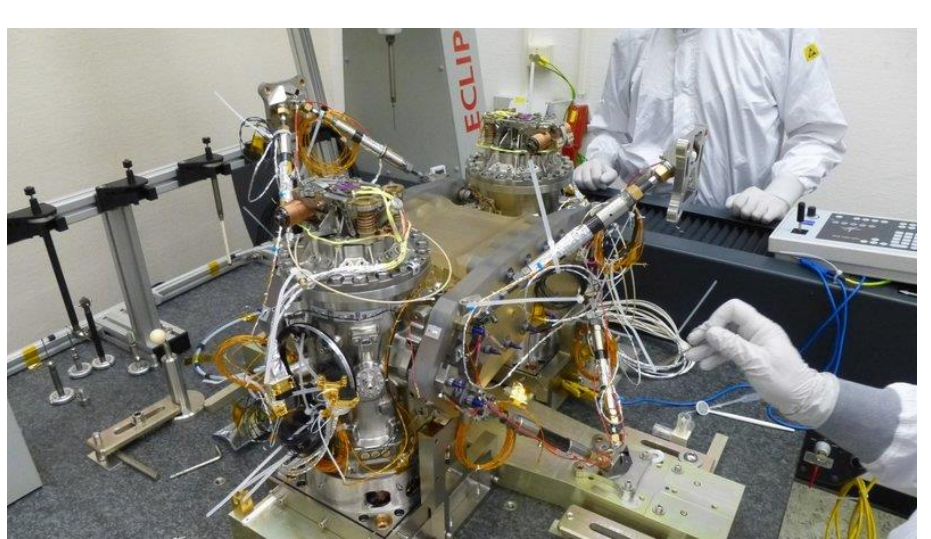

Linda Mondin | ICSO 2018-Chania | 09/10/2018 | Slide 29 


\section{ESA Developments}

- MO Alternatives - 3 Phase Contract, Parallel Competitive Phase 1 focussing on performance demonstration, single Phase 2/3 - LH EM build and test, TRL 6 2021

ESA Contract 1: COTS Approach

ESA Contract 2: Proprietary Approach

- RIO (US) ECDL as MO + fibre PA

- ECDL + resonant optical feedback as MO +

- RIO MO flown on US missions, NASA no SOA preamplifier + fibre power amplifier longer considering for LISA due to several technical and performance issues

- ESA assessment ongoing, assessment of performance versus LISA requirements

- MO delta development of $780 \mathrm{~nm}$ device demonstrated in rocket and drop tower quantum physics experiments

Down selection to one contractor for EM build and test in Q4 2018 


\section{ESA Developments}

口 PA + LEM

- Single industrial contract, 3 Phases, currently in Phase 2 EM build and test in laboratory environment, Phase 3 EM test in relevant environment

- Development assumes TESAT NPRO as MO, build/test EM of PA + LEM

- TRL 6 for EM PA + LEM 2020

$\square$ PA

- Two parallel contracts, developed and tested in concert with MO alternatives
MO
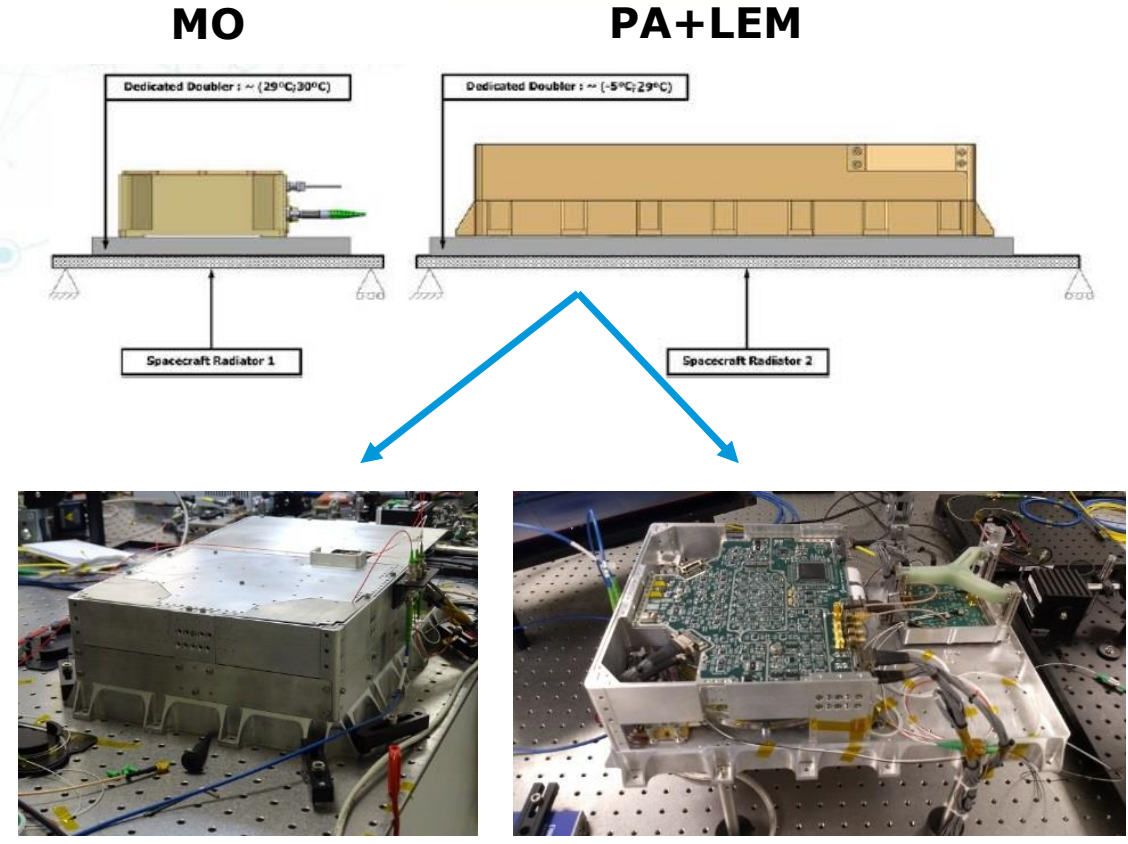


\section{ESA Developments}

\section{$\square$ Laser Pre-Stabilisation}

- European frequency reference system under development for Earth Observation potential future "Next Generation Gravitation Mission"

- NPL UK Cubic Cavity demonstrates performance required for LISA and is most mature European option

- Current TRL = 4/5

- Follow on activity required to demonstrate environmental robustness, TRL 6 expected 2019

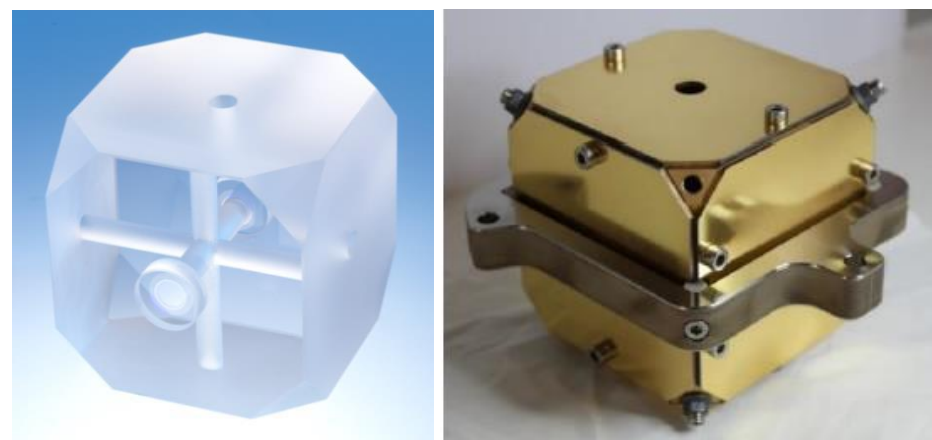

Images courtesy NPL C 


\section{Summary}

- Einstein predicted GW 100years ago, it took until 14 September 2015 before they were directly measured for the 1 st time.

- Einstein theory proven... and a new astronomy was born

- Multi-messenger astronomy started OCT-2017

- LISA PF has shown that LISA specifications are not impossible

$\square$ Launch is still far in the future but all LISA teams have been, and are, very busy designing the mission

$\square$ Laser System is at the core of LISA for metrology and telecommunication needs.

$\square$ Need to bring all LA sub-systems to TRL6 by mission adoption

$\rightarrow$ Activities on-track to reach this aim!

http://sci.esa.int/lisa/ 


\section{Thank you}

That's all folks! 